\title{
Evaluation of different ridge preservation techniques with a bovine xenograft by quantitative dimensional analysis - prospective, randomized clinical trial
}

\section{$+\frac{100}{20}$}

IMPLANT THERAPY OUTCOMES, SURGICAL ASPECTS

Kai R. Fischer, Dr.med.dent. ${ }^{1}$, Sven Mühlemann, Dr.med.dent. ${ }^{2}$, Ronald E. Jung, Prof. Dr.med.dent., PhD ${ }^{2}$, Anton Friedmann, Prof. Dr.med.dent. 1, Stefan Fickl, Priv.-Doz. Dr.med.dent. ${ }^{3}$

${ }^{1}$ Department of Periodontology, Faculty of Health, Witten/Herdecke University, Germany

${ }^{2}$ Clinic of Fixed and Removable Prosthodontics, Zuerich University, Switzerland

3 Department of Periodontology, Julius-Maximilians-University Wuerzburg, Germany

\section{Background and Aim}

The surgical removal of teeth is usually accompanied by significant changes in the hard and soft tissues of the alveolar ridge contour. Morphological changes occurring after tooth extraction have been well documented in literature. Schropp et al. evaluated 46 extraction sites in the upper and lower posterior areas and were able to demonstrate a shrinkage in the bucco-lingual ridge width of $50 \%$ after twelve months. Remarkably, two thirds of this change was to the buccal area. Subsequently, many studies have dealt with the reasons for these volumetric alterations. Loss of bony structure might impair the possibility of implant placement and create need for bone augmentation along or prior implant insertion. In addition, natural bony contour is crucial to an aesthetically pleasant implant treatment in the anterior region.

it was investigated whether immediate implant insertion is capable to stop these volumetric alterations. In contrast, studies of Araujo and coworkers showed that immediate implant placement in fresh extraction sockets resulted in the same or partially more bone resorption compared to untreated sites.

Consequently, multiple ridge preservation techniques - comparing various bone grafts with and without membrane application - have been proposed. Studies dealing with the augmentation of the extraction socket showed conflicting results and bone formation might even be delayed. Most histologic human studies have shown, however, that a large percentage of the augmented extraction socket presented bone substitute particles encapsulated in connective tissue especially in the coronal aspect.

Subsequently, we sought to assess the volumetric buccal ridge alterations following different ridge preservation techniques.

\section{Methods and Materials}

In this prospective randomized controlled clinical trial, 40 patients were included with one hopeless single-rooted tooth (incisors to premolars) resulting in one extraction site each. After tooth extraction each site was randomly assigned to one of four different treatment modalities (each group $\mathrm{n}=10$ )

Subjects were recruited consecutively by independent examiners at the Julius-Maximilians-University Würzburg, Germany. The University's Ethical Committee approved the consent form and research protocol on 31.10.2011 (183/11). Teeth with deficient buccal bone plates after tooth extraction were not excluded in this trial.

The following exclusion criteria were applied:

Subjects $<18$ years

Uncontrolled, manifest diabetes mellitus (based on patient's self report)

Status after radiation, chemotherapy or intravenous bisphosphonates within the last 5 years (based on patient's self report)

- Infectious diseases (HIV, Hepatitis B or C) (based on patient's self report)

- Pregnant or lactating female participants (based on patient's self report)

- Severe smokers (> 10 cigarettes/day) (based on patient's self report)

Multiple neighbouring extraction sites

- Untreated periodontal disease (probing depths $\geq 4 \mathrm{~mm}$ in more than 5 sites)

After minimal invasive tooth removal, the sockets were randomly scheduled to one of four treatment modalities:

Tx1 placement of a deproteinized bovine bone mineral (DBBM; Endobon ${ }^{\circledR}$, ZimmerBiomet, Carlsbad, USA) covered with a soft tissue punch from the palate;

Tx2 placement of DBBM alone;

Tx3 placement of DBBM covered with a resorbable collagen membrane (Osseoguard $^{\circledR}$, ZimmerBiomet);

Tx4 no additional treatment

The pre-extraction and follow-up impressions after 6 months were poured in dental stone (Tewerock ${ }^{\circledR}$, Kettenbach, Eschenburg, Germany). Thereafter, the master casts were optically scanned with a laboratory optical scanner (ceramill ${ }^{\circledR}$ p400, Amann Girbach, Pforzheim, Germany) resulting in digital STL files (Standard Tessellation Language). For each patient the digital surface models representing the two study time points were imported into the volume analysis software (Swissmeda/SMOP, Zürich, Switzerland). The bestfit algorithm was used to superimpose Zuich, swizerland). The best it algorithm was use (Fig. 1-4).
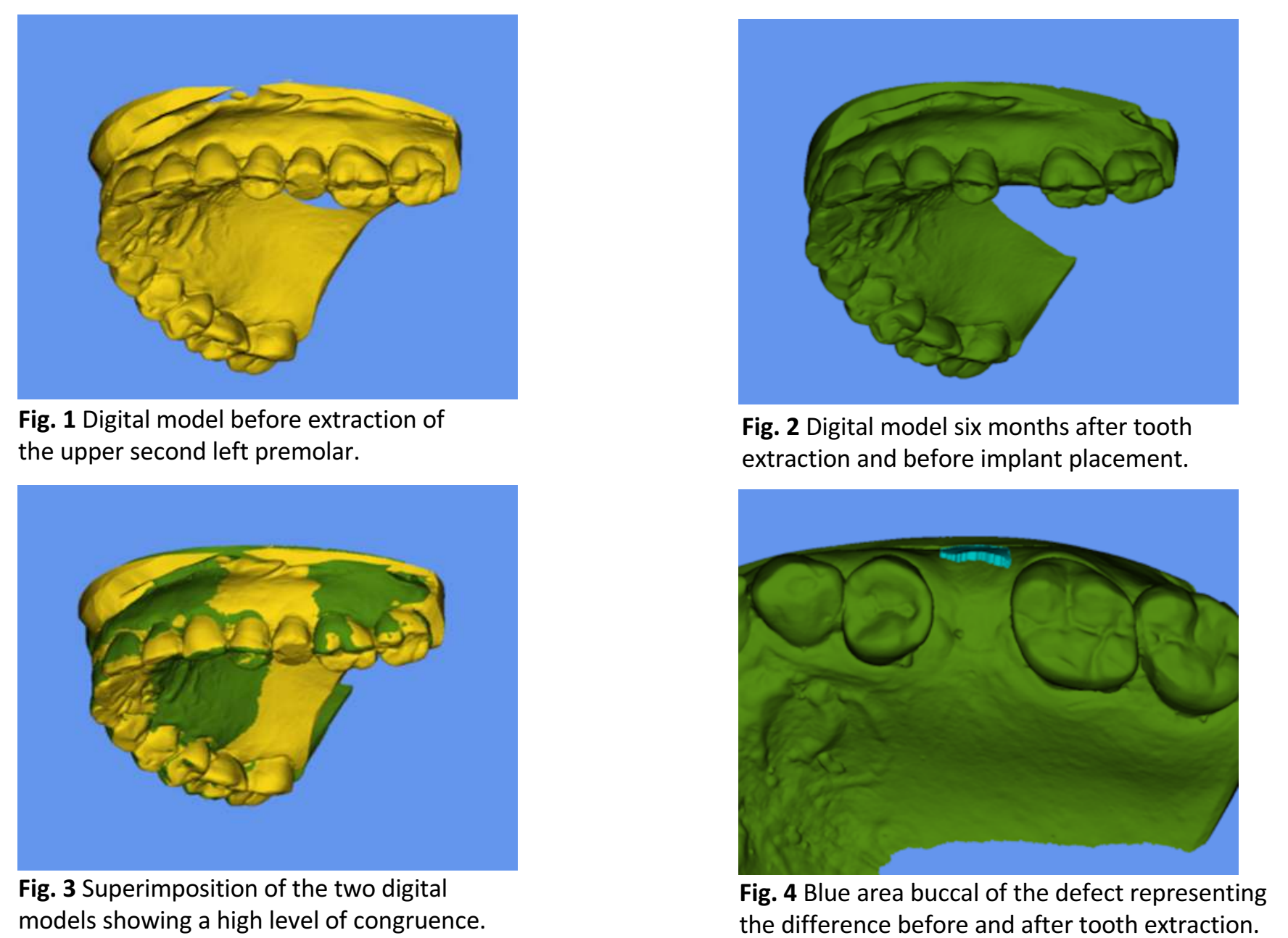

\section{Results}

The study population consisted of 40 patients (24 female and 16 male) aged 18 to 80 years (mean age $55.7 \pm 14.85$ years) with one or more hopeless teeth with intact neighbouring teeth. 35 patients completed the study presenting 35 extraction sockets and complied with all study appointments. No statistical significant difference was found between the selected and evaluated surface area comparing the four different groups (Kruskal-Wallis Test: $p>0.05$ ).

The three socket preservation techniques ( $T \times 1-T \times 3)$ resulted in similar buccal contour changes six months after tooth extraction with the smallest changes in Tx1. The control group (Tx4) showed twice the volumetric change compared to socket preservation techniques (Table 1). This difference did not reach statistical significance (Kruskal-Wallis Test for comparison of all groups: $p>0.05$; Mann-Whitney $U$ Test for comparison between single groups: Tx1-Tx3 vs Tx4, p > 0.05; Fig. 5).

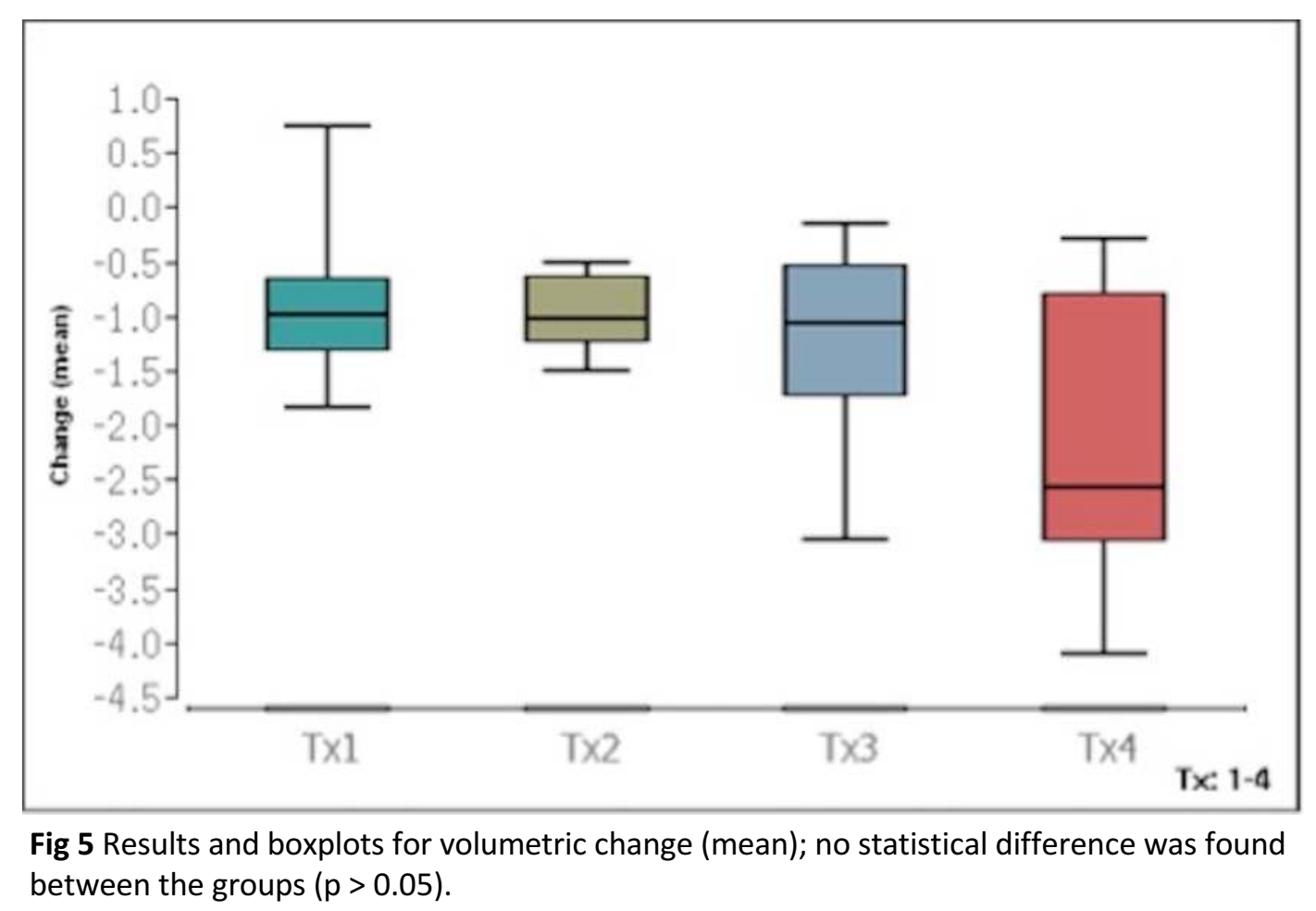

\section{Conclusions}

In this randomized controlled clinical trial, we tried to identify whether ridge preservation with a bovine xenograft bone substitute reduces alveolar ridge contour changes after tooth extraction and whether these procedures facilitate implant placement and reduce further need for bone augmentation. No statistical significant difference was found between the four treatment modalities. Nevertheless, the three socket preservation techniques resulted in similarly limited buccal contour changes $(<1 \mathrm{~mm})$ with the smallest changes occurring in the treatment group applying a soft tissue punch. Leaving the extraction socket solely to blood clot formation (Tx4, controls) did result in twice as much contour loss (>2 $\mathrm{mm}$ ) with the highest variance. This tissue loss might be clinical relevant especially in aesthetically demanding cases, respectively. Still, the clinical importance of this finding is unclear, as drill condition and the necessity of re-augmentation to be able to place an implant were not significantly different between the treatment groups.

\section{References}

Ten Heggeler JM, Slot DE and Van der Weijden GA (2011) Effect of socket preservation
therapies following tooth extraction in non-molar regions in humans: a systematic review. Clin Oral Implants Fickl S, Zuhr O, Wachtel H, Kebschull M and Hurzeler MB (2009) Hard tissue alterations
after socket preservation with additional buccal overbuilding: a study in the beagle dog. $J$
Clin Periodontol 36:898-904. Schropp $L$, Wenzel A, Kostopoulos $L$ and Karring T $T$ (2003) Bone healing and soft tissue
contour changes following single-tooth extraction: a clinical and radiographic 12-month
prospective study lnt $J$ Periodontics Restorative Dent 23.313-23 Carmagnola $D_{2}$ Adriaens $P$ and Berglundh T (2003) Healing of human extraction sockets
filled with Bio-Oss. Clin Oral Implants Res 14:137-43. 